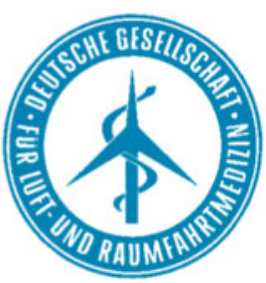

Mitteilungen der

Deutschen Gesellschaft für

Luft- und Raumfahrtmedizin e. V. Nr. 5/2016

DGLRM-Homepage: www.dglrm.de

Geschäftsstelle der DGLRM

Frank Teichert

Postfach 870204

13162 Berlin

E-Mail: Geschaeftsstelle@dglrm.de

Tel.: 0176/5624-2876

\section{Wir gratulieren}

- 65 Jahre, Dr. Wolfgang Schardt, Sankt Augustin, 04.10.1951

- 80 Jahre, Dr. Toni Kressin, Berlin, 09.10.1936

Wir begrüßen als neue Mitglieder

- Frau Dr. Lida Prieß aus Münster

- Herrn Dr. Walter Heimbach aus Köln

\title{
Sehr geehrte Damen und Herren, liebe Mitglieder der DGLRM,
}

dieser September hatte es wirklich in sich. Er begann mit dem erstmals unter der Ägide der DGLRM gelaufenen „Basic Course" der Deutschen Akademie für Flug- und Reisemedizin (DAF) in Frankfurt, war im Weiteren mit Arbeiten zur Finalisierung des Konzeptentwurfs des Vorstands unserer Gesellschaft zur zukünftigen Gestaltung der DAF gGmbH sowie mit der 5. European Conference of Aviation Medicine (ECAM) in Oslo und schließlich mit letzten Vorbereitungen und der Durchführung unserer eigenen Jahrestagung in Bückeburg ausgefüllt.

\section{Erster Basis Kurs unter der Ägide der DGLRM erfolgreich gelaufen}

Der sogenannte „Basic Course Aviation Medicine“, in dem die nötigen Grundkenntnisse, die als Voraussetzung für eine Tätigkeit als flugmedizinischer Sachverständiger beziehungsweise Authorized Medical Examiner (AME) gemäß MED.D.020 der VO(EU) 1178/2011 erforderlich sind, vermittelt werden, lief vom 3. bis zum 11. September 2016 in den Räumen des Medizinischen Dienstes der Lufthansa in Frankfurt am Main. Herr OTA PD Dr. Weber vom Zentrum für Luft- und Raumfahrtmedizin der Luftwaffe hatte sich freundlicherweise bereit erklärt, die Lehrgangsleitung zu übernehmen und hat diesen Kurs über die gesamte Zeit kompetent geführt und betreut. Als Präsidentin der DGLRM habe ich es mir nicht nehmen lassen, die Teilnehmer, die aus insgesamt 7 Nationen (Deutschland, Belgien, Österreich, Schweiz, Schweden, Tschechien und den USA) kamen, zu begrüßen. Gleichzeitig konnte ich mich vor Ort von der sorgfältigen und hervorragenden Vorbereitung, die, wie bereits in vergangenen Jahren, in den bewährten Händen von Frau Froese lag, überzeugen. Ohne Frage war, neben der eigentlichen Wissensvermittlung, der von Herrn Prof. Stüben organisierte Flugtag, bei dem die Teilnehmer eigene Erfahrungen beim Motor- und Segelflug (Windenstarts, FSchlepp, Motorsegler), aber auch im Segelkunstflug und im Ballon fahren machen konnten, einer der absoluten Höhepunkte des Lehrgangs. Für den Lehrgangsleiter war natürlich die Tatsache, dass alle
32 Teilnehmer ohne Ausnahme den Abschlusstest erfolgreich absolviert haben, eine schöne Bestätigung der Qualität der Wissensvermittlung während des Kurses (Abb. 1-3).

An dieser Stelle möchte ich allen DGLRMMitgliedern, die, mit ihrer Bereitschaft, ihrem persönlichen Einsatz und ihrer tatkräftigen Mitarbeit und Unterstützung während der Vorbereitung und Durchführung, diesen Kurs überhaupt erst möglich gemacht haben, danken. Im Besonderen gilt dieser Dank Herrn OFA Dr. Reichert vom Zentrum für Luft- und Raumfahrtmedizin der Luftwaffe, der mir bei der Erarbeitung der Unterlagen für den Lizenzierungsprozess beim LBA eine wertvolle Stütze war. Ebenso zu Dank verpflichtet fühle ich mich dem jetzigen Geschäftsführer Herrn Eckard Glaser, dem Kursleiter Herrn OTA PD Dr. Weber und allen Referenten sowie Frau Froese, der guten Seele des Kurses.

\section{Die ESAM wurde 10 Jahre alt} und hat dieses Jubiläum würdig begangen

Die European Society of Aerospace Medicine (ESAM) hat vom 15. bis 18. September im Homenkollen Parkhotel in Oslo ihren 5. European Congress of Aerospace Medicine (ECAM) durchgeführt. Dieser wurde in Zusammenarbeit der ESAM mit der Norwegischen Gesellschaft für Flugmedizin und gleichzeitig auch erstmals als ein gemeinsamer Kongress der ESAM in Zusammenarbeit mit der Aerospace Medical Association (AsMA) in Europa durchgeführt. Zunächst als Versuch gedacht, war dies ein voller Erfolg. Die 413 Teilnehmer haben die Erwartungen der Organisatoren bei Weitem überschritten und so dazu beigetragen, das 10-jährige Bestehen der ESAM angemessen zu begehen.

Zur Eröffnung des Kongresses hat die Bürgermeisterin der Stadt Oslo zu einem feierlichen Empfang ins Rathaus eingeladen. Hier konnten die Teilnehmer auf einer geführten Tour durch das Rathaus auch den Saal, in dem jedes Jahr am Todestag Alfred Nobels, dem 10. Dezember, der Friedensnobelpreis vergeben wird, bewundern (Abb. 4). 
Der eigentliche Kongress, der von Freitag bis Sonntagmittag stattfand, stand unter dem Motto „Pushing the Limits of Aerospace Medicine together“. Auf ihm haben sich Experten der ganzen Welt über Themen wie safety management, pilot performance, unmanned aerial vehicel operations, Aspekte extremer Umwelten sowie über das große Thema „Mental Health“ und Möglichkeiten, die Flugsicherheit zu erhöhen, unterhalten. Außerdem wurden Herausforderungen und Chancen regulativer Eingriffe in die flugmedizinische Begutachtung der Zukunft beleuchtet. Die deutsche Delegation hat sich auf diesem Kongress sehr gut ins Licht setzen können. Im Wettbewerb um den besten Vortrag und das beste Poster wurden der Vortrag von Oberstarzt Dr. Grell und das Poster von Oberfeldarzt Dr. Schwerer, beide vom Zentrum für Luft- und Raumfahrtmedizin der Luftwaffe, von der Jury mit einem zweiten Platz bedacht.

Auf der General Assembly, der Mitgliederversammlung der ESAM, wurde in diesem Jahr ein neues Executive Committee (EC=Vorstand) gewählt und der im vergangenen Jahr gewählte sogenannte „president elect“ Dr. Anthony Wagstaff aus Norwegen in sein Amt als Präsident eingeführt (Abb. 5). Deutschland konnte wiederum einen Vertreter in den Vorstand entsenden. Herr Prof. Hinkelbein wird als Schatzmeister der ESAM fungieren. Außerdem wurde ich selbst, aufgrund meiner Vorerfahrung als frühere Schatzmeisterin der ESAM, zur Kassenprüferin gewählt.

Das Galadinner am Sonnabend war dann ein ganz besonderer Höhepunkt dieser Veranstaltung (Abb. 6). Dazu trug nicht nur der außerordentlich schöne Saal bei. Aus Anlass des 10-jährigen Jubiläums wurden ein Rückblick auf die Entwicklung der ESAM gewagt und besondere Verdienste Einzelner in diesem Prozess hervorgehoben. So erhielt der erste Präsident der ESAM, Dr. Roland Vermeiren, die erstmals vergebene Auszeichnung als Chevalier der ESAM (Abb. 7).

Insgesamt ist die Historie dieser europäischen Organisation eine Erfolgsgeschichte. Die ESAM begann 2006, wollte als paneuropäisches unabhängiges Forum die Sicherheit und die Gesundheit aller in der Luft- und Raumfahrt tätigen Operateure stärken sowie die europäischen luft- und raumfahrtmedizinischen Interessen über die nationalen

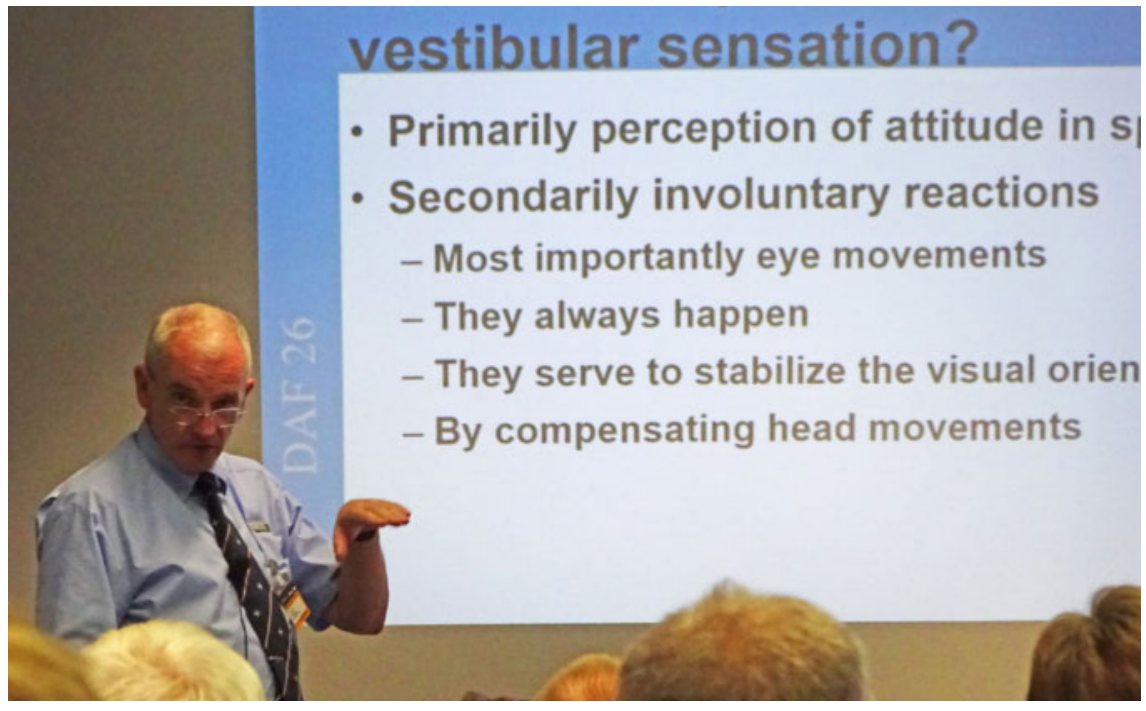

Abb. 1 Bei der Unterweisung von Orientierung und Desorientierung durch Dr. Wetzig.

Quelle: PD Dr. Carla Ledderhos

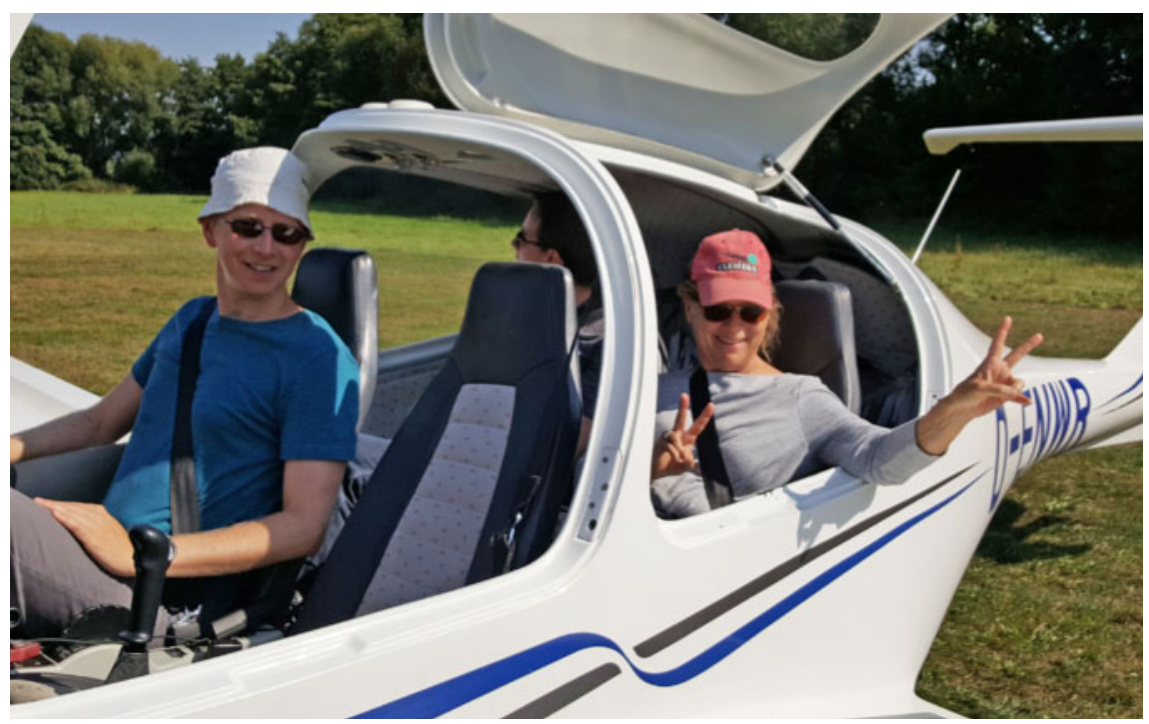

Abb. 2 Impressionen vom Flugtag.

Quelle: Dr. Constance Winslow

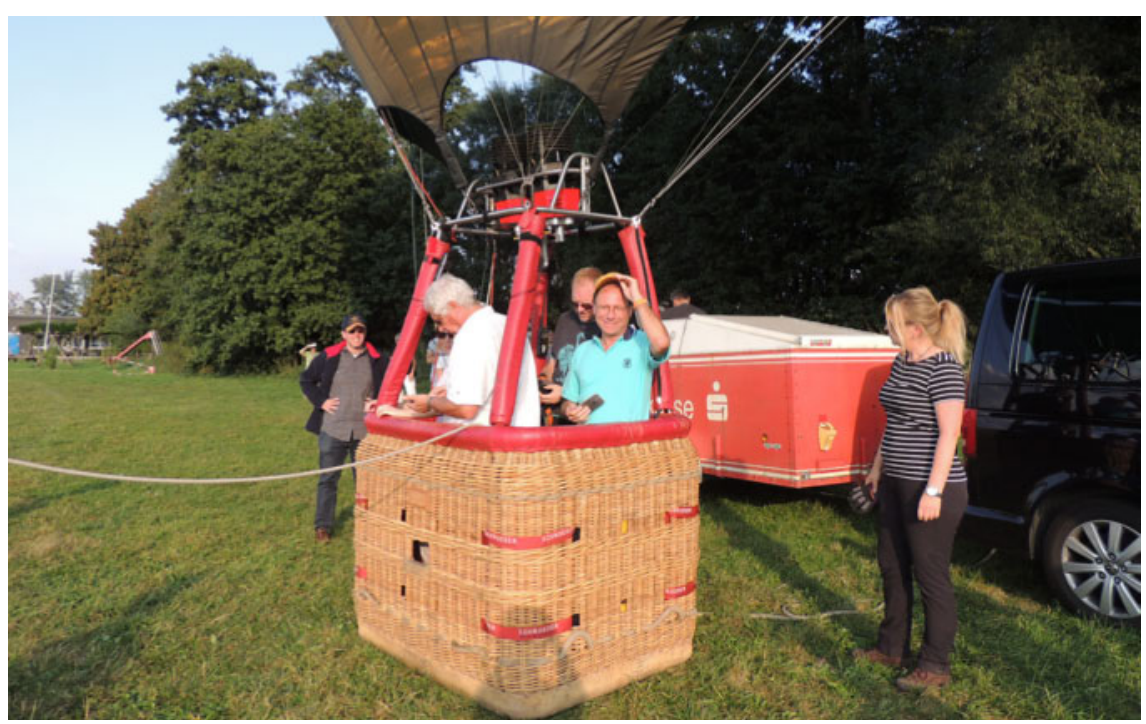

Abb. 3 Impressionen vom Flugtag. 


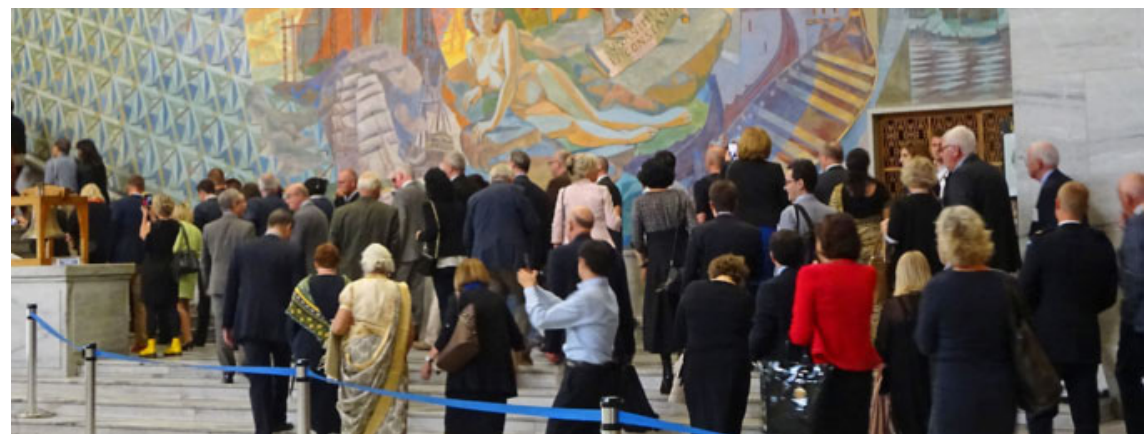

Abb. 4 Die Teilnehmer der ECAM 5 auf dem Weg zum Empfang im Rathaus von Oslo.

Quelle: PD Dr. Carla Ledderhos

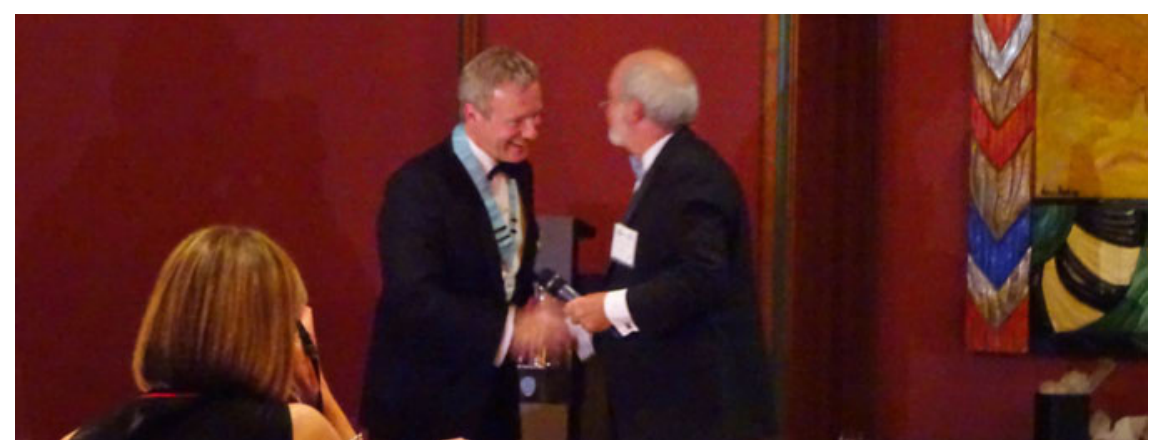

Abb. 5 Übergabe der Amtskette vom alten Präsidenten, Dr. Kevin Herbert, an den neuen Präsidenten der ESAM, Dr. Anthony Wagstaff, auf dem Gesellschaftsabend.

Quelle: PD Dr. Carla Ledderhos

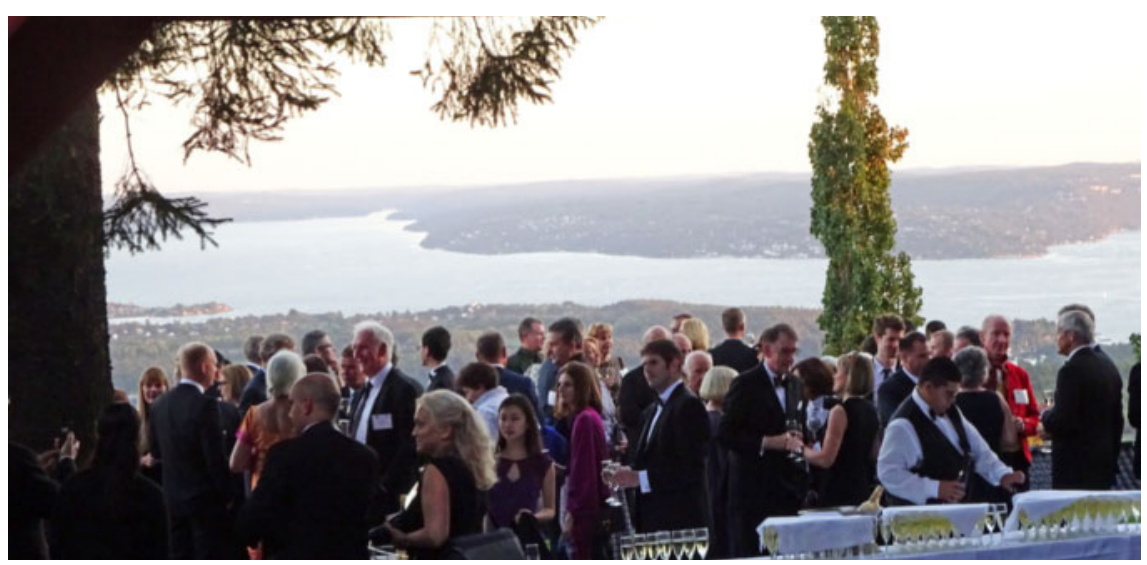

Abb. 6 Eröffnung des Abschlussabends im Hotel auf dem Holmenkollen in Oslo.

Quelle: PD Dr. Carla Ledderhos

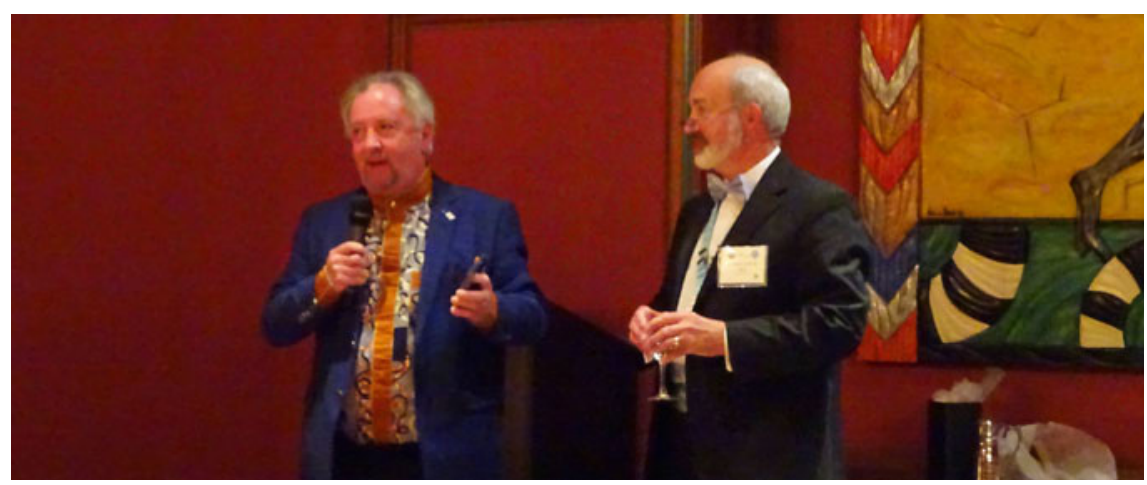

Abb. 7 Roland Vermeiren erhält für seine Verdienste die erste von der ESAM vergebene Chevalier Medal. Quelle: PD Dr. Carla Ledderhos und organisatorischen Grenzen hinaus bündeln. Inzwischen sind fast alle europäischen Länder in ihr vertreten. Zählt man alle Mitglieder der einzelnen Gesellschaften der verschiedenen Staaten zusammen, so ist sie schon heute die zahlenmäßig größte flugmedizinisch orientierte Gesellschaft der Welt. Nach nur 10 Jahren ihres Bestehens ist aus dem empfindlichen Pflänzchen von einst inzwischen eine junge Pflanze geworden. Deutschland ist stolz darauf, zu den Gründungsmitgliedern dieser Gesellschaft zu gehören, der wir für ihre Teenagerjahre viel Erfolg und ein weiteres Wachstum wünschen!

\section{DGLRM Jahrestagung 2016 \\ in Bückeburg}

Der Jahreskongress unserer Gesellschaft war in diesem Jahr durch ein umfangreiches Programm gekennzeichnet. Dies betraf nicht nur die wissenschaftlichen und Weiterbildungsveranstaltungen, sondern auch die Gremiensitzungen und die Mitgliederversammlung, in denen eine Vielzahl von Gesprächsthemen zu bearbeiten war.

Die Plenarvorträge sowie die Fachbesichtigungen im Internationalen Hubschrauberausbildungszentrum der Bundeswehr und im Hubschraubermuseum aber auch die Nachtwächterführung durch Bückeburg haben dabei für die notwendige Balance zwischen Spannung und Entspannung gesorgt. Das Hubschraubermuseum war nicht nur ein würdiges Ambiente, sein Team stand uns auch bei allen Fragen helfend zur Seite und die Cateringfirma hat uns aufs Beste verwöhnt. Die Tagungsteilnehmer haben sich sehr lobend über die gute Betreuung durch Herrn und Frau Bals sowie durch Herrn OTL a.D. Mallwitz vom Hubschraubermuseum geäußert. Dieses Lob leite ich gerne weiter.

Erneut gab uns diese Tagung auch die Möglichkeit, mit den Verantwortlichen für die Flugmedizin im zuständigen Referat des Bundesministeriums für Verkehr und digitale Intrastruktur ins Gespräch zu kommen. Der Vortrag von Herrn Kamp war für alle Teilnehmer eine wichtige Informationsquelle für zu erwartende Änderungen in unserem Gebiet. Ich denke ich spreche hier im Interesse aller Mitglieder, wenn wir uns als Vorstand bemühen werden, diese Kontakte weiter zu pflegen und auszubauen. 


\section{Posterpreise 2016}

Auch in diesem Jahr wurden auf der Tagung wieder Posterpreise verliehen. Eine 12-köpfige Kommission unter der Leitung von OTA a.D. Prof. Hans Pongratz hat nach zuvor klar definierten Kriterien ihre Urteile gefällt. In diesem Jahr konnten insbesondere Arbeiten aus dem Zentrum für Luft- und Raumfahrtmedizin der Luftwaffe die Juroren überzeugen.

Die Posterpreisträger 2016:

1. Pippig $\mathrm{T}$

Prädiskotische Veränderungen an der Hals- und Lendenwirbelsäule bei asymptomatischen Bewerbern für eine fliegerische Verwendung in der Bundeswehr. Häufigkeit und Begutachtung.

2. Ledderhos $\mathrm{C}$

Anti-G-Schutz im Wandel der Zeiten - Meilensteine der Entwicklung von Anti-G-Anzügen.

3. Schwerer M und Graw M Sportflugzeugunfälle im Einzugsgebiet der Rechtsmedizin München im ersten Halbjahr 2016.

Daneben wurden 2 Preise für junge FirstPresenter vergeben. Sie gingen an Frau Herzog, die sich dem Thema „Effekte einer Sprachführung in mobilen Navigationsapplikationen auf das räumliche Situationsbewusstsein, die Flugpräzision und subjektive Workload von Piloten in der allgemeinen Luftfahrt" angenommen hat und an Herrn Wahnschaffe, der die Mitgliederstruktur der Deutschen Gesellschaft für Luft- und Raumfahrtmedizin (DGLRM) nach wissenschaftlichen Gesichtspunkten analysiert hat (Abb. 8). Wir hoffen, dass diese Preise ein kleiner Ansporn für junge Nachwuchswissenschaftler sein werden, sich in der Flugmedizin zu engagieren.

Mitgliederversammlung der DGLRM

Die diesjährige Jahreshauptversammlung unserer Gesellschaft (Abb. 9) war so gut besucht wie schon seit Jahren nicht mehr und stand vor allem im Zeichen der Neuwahlen zum Vorstand und Vorstandsrat sowie von Diskussionen über die zukünftige Gestaltung der zivilen flugmedizinischen Ausbildung in Deutschland und der Rolle, die die DGLRM dabei einnehmen soll. Daneben standen die Rechenschaftslegung des Vorstands und der Arbeitsgruppenleiter über die im letzten Jahr geleistete Arbeit sowie ein Beschluss zur Erhöhung der Mitgliedsbeiträge ab dem

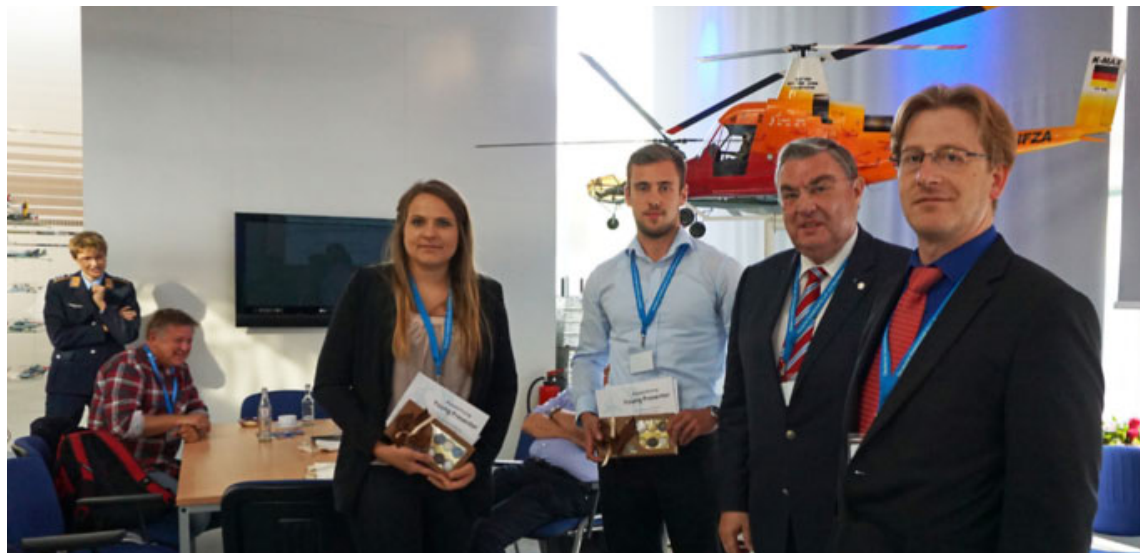

Abb. 8 Vergabe der First-Presenter-Preise an junge Wissenschaftler.

Quelle: Gens/Teichert

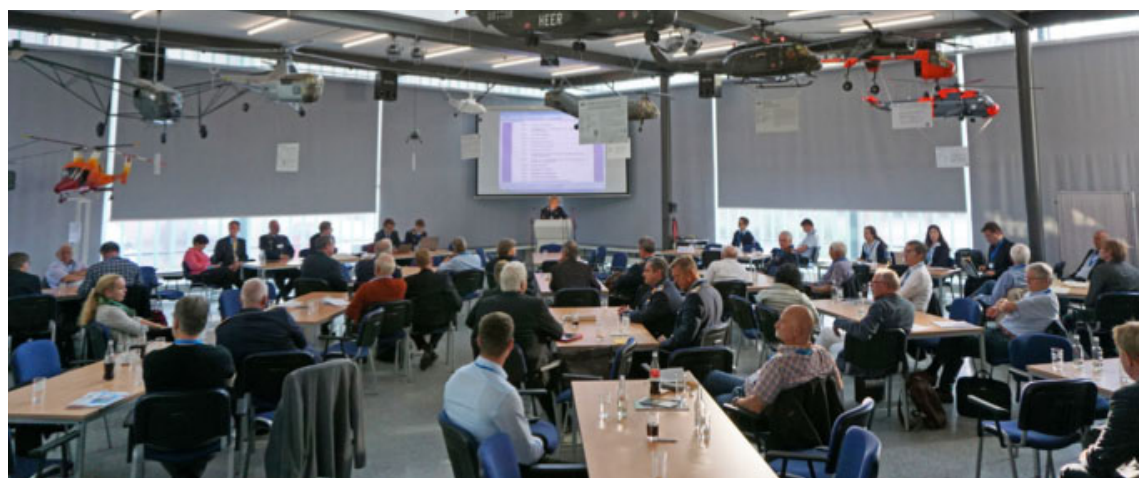

Abb. 9 Blick in die Mitgliederversammlung der DGLRM.

Quelle: Gens/Teichert

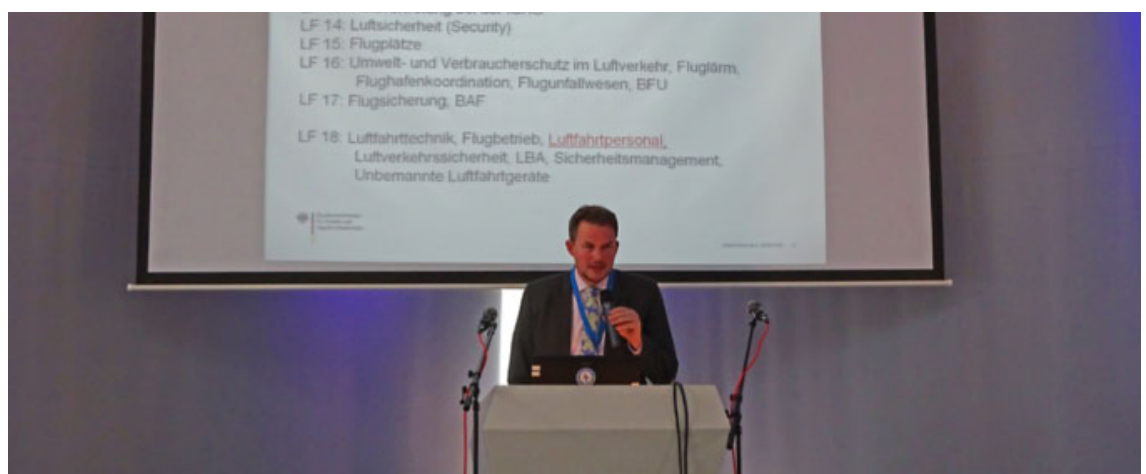

Abb. 10 Herr Kamp vom zuständigen Referat im Bundesministerium für Verkehr und digitale Infrastruktur (BMVI) bei seinem Vortrag.

Quelle: PD Dr. Carla Ledderhos

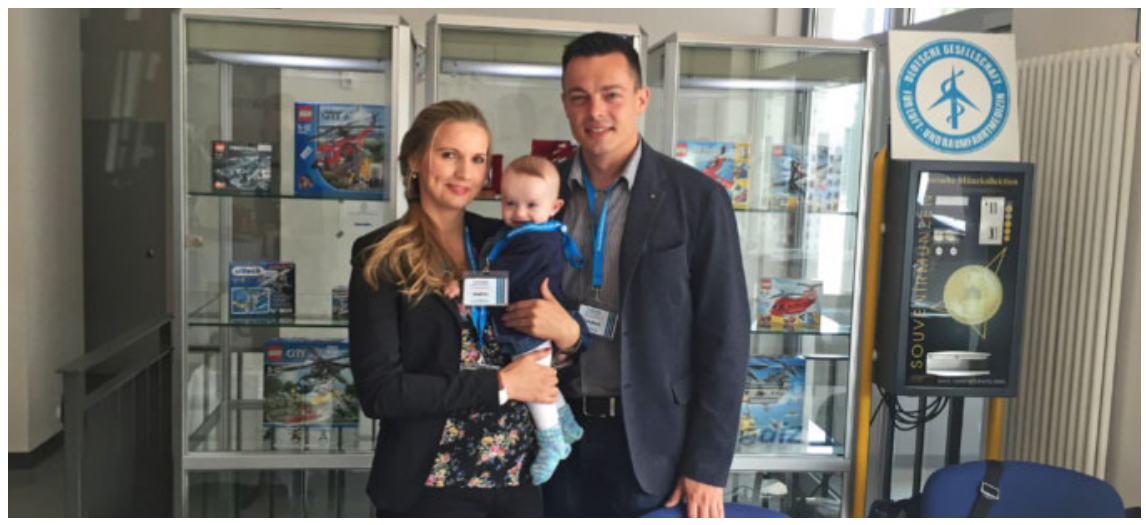

Abb. 11 Unser Organisationsteam - Wir beginnen schon sehr früh mit der Nachwuchsgewinnung. Quelle: Gens/Teichert 
nächsten Jahr von 100 auf 120 Euro im Mittelpunkt der Veranstaltung.

Mit ihrer jetzigen Rolle als Alleingesellschafter der DAF gGmbH sind für die DGLRM viele Pflichten aber auch Chancen verbunden, denen wir uns stellen wollen und müssen.

Im Vorfeld zu unserer Mitgliederversammlung hatte der Vorstand ein Konzeptpapier erarbeitet, es allen Mitgliedern zur Kenntnis gegeben, intensive Diskussionen mit dem Vorstandsrat geführt und dabei versucht, die bestehenden divergierenden Lösungsauffassungen einer einheitlichen, tragfähigen Lösung zuzuführen. Im Ergebnis dieser Gespräche haben sich Vorstand und Vorstandsrat auf ein gemeinsames weiteres Vorgehen geeinigt, das der Mitgliederversammlung zur Abstimmung unterbreitet und dort einstimmig angenommen wurde. Alle Mitglieder, insbesondere aber diejenigen, die diese Jahrestagung nicht besuchen konnten, finden das Konzeptpapier, die auf der ordentlichen Mitgliederversammlung dargebotene begründende Präsentation sowie den genauen Wortlaut des Beschlusses im Mitgliederbereich unserer Homepage www.dglrm.de.

In Sachen Deutsche Akademie für Flugund Reisemedizin geht es nun darum, den Refresher-Kurs, der vom 4. bis 6. November in Seeheim stattfinden wird, mit interessanten Vorträgen auszufüllen und die Lizensierung des „Advanced Kurses“ durch das LBA erfolgreich abzuschließen. Inzwischen konnten alle Referenten gewonnen werden und sehen den Kursen mit Interesse entgegen.

Daneben wird der Vorstand mit der Umsetzung der Beschlüsse der Mitgliederversammlung beginnen.

\section{Wahlen zum neuen Vorstand}

Entsprechend der Tradition in unserer Gesellschaft folgt einer Legislaturperiode unter der Leitung eines Angehörigen der Bundeswehr eine, in der ein Kollege aus dem zivilen Bereich die Leitung der Gesellschaft übernimmt. Zum neuen Präsidenten der DGLRM, der ab Januar 2017 die Geschicke unserer Gesellschaft lenken wird, wurde Prof. Dr. Hinkelbein gewählt. Dem geschäftsführenden Vorstand werden dann OTA Dr. Pippig als erster Vizepräsident, meine Person als zweiter Vizepräsident und Herr Dr. Neuhaus als Schatzmeister angehören. Für die Tätigkeit als Sekretär hat Frau Gammel das Votum der Mitglieder erhalten.
Zu Beisitzern sind Frau Dr. Stern, Herr Glaser sowie Herr Prof. Ullrich und Herr Dr. Siedenburg gewählt worden. Wir alle wünschen dem neuen Vorstand viel Kraft bei der Bewältigung der anstehenden Aufgaben.

Die diesjährigen Wahlen zum Vorstand unserer Gesellschaft wurden entsprechend der 2013 verabschiedeten Satzung nach einem neuen Modus durchgeführt. Der eigentliche Wahlprozess entpuppte sich dabei als sehr zeitaufwendig und mitunter unübersichtlich. Es gab am Ende eine Reihe von Stimmen, die angeregt haben, dieses Prozedere bei einer möglichen erneuten Satzungsänderung noch einmal kritisch zu hinterfragen.

\section{Vorstandsratswahlen}

Bei der Wahl zum Vorstandsrat haben sich folgende Kolleginnen und Kollegen bereit erklärt, dem Vorstand in seiner nächsten Legislaturperiode beratend zur Seite zu stehen: Dr. Frank, Dipl. Ing. Gens, Dr. Harsch, Dr. Hedtmann, Dr. Janicke, Dr. Kimmich, Herr Knüppel, Prof. Kriebel, Prof. Landgraf, Dr. Mohtadi, Prof. Pongratz, Prof. Schick, Dr. Sigfrid, Dr. Thiel, Dr. Trammer, Dr. Welsch, Dr. Werner, Dr. Wurster. In seiner konstituierenden Versammlung hat der neue Vorstandsrat, der am 1. Januar 2017 seine Arbeit aufnehmen wird, Herrn Dr. Hedtmann zum Vorstandsratssprecher gewählt.

Weitere Informationen zu unserer Jahrestagung sowie die Vorträge (soweit von den Autoren freigegeben) und Bilder erhalten Sie auf unserer Homepage. Ich wünsche Ihnen schon jetzt viel Spaß beim Erkunden.

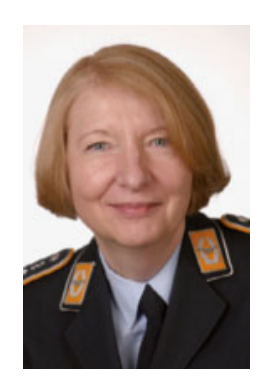

Ihre

\section{Kongresse 2016}

$64^{\text {th }}$ International Congress of Aviation and Space Medicine (ICASM)

Int. Academy of Aviation and Space Medicine

Termin: 06.11.-10.11.2016

Ort: Neu-Delhi, Indien

Internet: www.icasm.org
Verantwortlich für den Inhalt der DGLRM-Seiten

Deutsche Gesellschaft für Luft- und Raumfahrtmedizin e. V. (DGLRM)

OFA PD Dr. Carla Ledderhos, Präsidentin (V.i.S.d.P.)

Zentrum für Luft- und Raumfahrtmedizin der Luftwaffe

Str. der Luftwaffe 322, 82242 Fürstenfeldbruck CarlaLedderhos@bundeswehr.org

Bankverbindung

Deutsche Bank Hamburg

Konto-Nr.: 388948200 , BLZ: 20070000

IBAN: DE 63200700240388948200

BIC: DEUTDEDBHAM

Änderungen für die Mitgliederkartei bitte an: Dipl. Ing. Christine Gammel

Zentrum für Luft- und Raumfahrtmedizin der Luftwaffe

Str. der Luftwaffe 322, 82242 Fürstenfeldbruck

ChristineGammel@bundeswehr.org

\section{Lehrgänge 2016/2017}

24. Refresher-Seminar Flugmedizin

(deutsch)

Deutsche Akademie für Flug- und Reisemedizin $\mathrm{GmbH}$

Termin: 04.11.-06.11.2016

Ort: $\quad$ Lufthansa Training \&

Conference Center, SeeheimJugenheim

Kontakt: Sigrid Froese, Tel: +49(0)69/69691222

Internet: www.flugmed.org/ www.eusam.org

EUSAM - Advanced course 26 (englisch) European School of Aviation Medicine Termin: 03.12.-11.12.2016

Ausgebucht, Warteliste

Ort: Medizinischer Dienst der Deutschen Lufthansa auf der Lufthansa-Basis, Flughafen Frankfurt

Kontakt: Sigrid Froese Tel: $+49(0) 69 / 69691222$

Internet: www.flugmed.org/ www.eusam.org

Fortbildung für fliegende Fliegerärzte in St. Auban

Leitung: Dr. Thomas Buchsein und Dr. Heiko Wassill

Termin: 20.03.-31.03.2017

Ort: $\quad$ CNVV, Centre National de Vol à Voile

Anmeldung unter: St. Auban@flug-med.de Internet: www.stagekriebel.de 